\title{
The cosmic web for density perturbations of various scales
}

\author{
I. Suhhonenko ${ }^{1}$, J. Einasto ${ }^{1,2,3}$, L. J. Liivamägi ${ }^{1}$, E. Saar ${ }^{1,2}$, M. Einasto ${ }^{1}$, G. Hütsi ${ }^{1}$, V. Müller ${ }^{4}$, A. A. Starobinsky ${ }^{5,6}$, \\ E. Tago ${ }^{1}$, and E. Tempel ${ }^{1}$ \\ 1 Tartu Observatory, 61602 Tõravere, Estonia \\ e-mail: ivan@aai.ee \\ 2 Estonian Academy of Sciences, 10130 Tallinn, Estonia \\ 3 ICRANet, Piazza della Repubblica 10, 65122 Pescara, Italy \\ 4 Astrophysical Institute Potsdam, An der Sternwarte 16, 14482 Potsdam, Germany \\ 5 Landau Institute for Theoretical Physics, Moscow 119334, Russia \\ ${ }^{6}$ Research Center for the Early Universe (RESCEU), Graduate School of Science, The University of Tokyo, Tokyo 113-0033, Japan
}

Received 23 December 2010 / Accepted 9 March 2011

\section{ABSTRACT}

\begin{abstract}
Aims. We follow the evolution of galaxy systems in numerical simulations. Our goal is to understand the role of density perturbations on various scales in the formation and evolution of the cosmic web.

Methods. We perform numerical simulations with the full power spectrum of perturbations, and with a spectrum cut at long wavelengths. In addition, we have one model, where we cut the intermediate waves. We analyse the density field and study void sizes and density field clusters in different models.

Results. Our analysis shows that the fine structure (groups and clusters of galaxies) are created by small-scale density perturbations of scale $\leq 8 h^{-1} \mathrm{Mpc}$. Filaments of galaxies and clusters are created by perturbations of intermediate scale from $\sim 8$ to $\sim 32 h^{-1} \mathrm{Mpc}$, and superclusters of galaxies by larger perturbations.

Conclusions. We conclude that the texture of the cosmic web is determined by density perturbations of the scales up to $\sim 100 h^{-1} \mathrm{Mpc}$. Larger perturbations do not change the texture of the web, but modulate the richness of galaxy systems, and make voids emptier.
\end{abstract}

Key words. large-scale structure of Universe - cosmology: miscellaneous - cosmology: theory - galaxies: clusters: general dark matter - methods: numerical

\section{Introduction}

The first studies of the three-dimensional distribution of galaxies and clusters of galaxies demonstrated that they are not distributed randomly, but are mostly located in filamentary superclusters connected by galaxy filaments to form a connected network, leaving large regions devoid of galaxies (Gregory \& Thompson 1978; Jõeveer \& Einasto 1978; Jõeveer et al. 1978; Tarenghi et al. 1978; Tully \& Fisher 1978; Einasto et al. 1980; Zeldovich et al. 1982). This observational picture was rather similar to the theoretical prediction of Zeldovich (1970), the so-called pancake scenario of galaxy formation, as discussed by Zeldovich (1978). However, there were some important differences between the models and the observations. In the real world, the most common structural elements were filaments of galaxies and their clusters, whereas the Zeldovich (1978) scenario predicted the formation of flat formless pancakelike systems (for a discussion see Zeldovich et al. 1982; and Einasto et al. 1983). The simulations performed for the neutrinodominated universe, called the hot dark matter (HDM) model (White et al. 1983), had similar problems.

The problem of the absence of the fine structure was solved when the cold dark matter (CDM) scenario was suggested by Bond et al. (1982) and others. The basic difference between the HDM and CDM scenarios is the absence or presence of small-scale density fluctuations. The comparison of simulations for both dark matter types demonstrated the advantages of the CDM model - in this model, the fine structure is present in the form of filaments of various scales (see Melott et al. 1983; and
White et al. 1987, among others). An even closer agreement of simulations with observations is obtained in models with a cosmological constant, $\Lambda \mathrm{CDM}$, as demonstrated by Gramann (1988).

One problem remained: both numerical simulations and direct observations suggested that the dominant structural elements of the cosmic web are filaments of various scales, whereas the classical Zeldovich (1970) scenario predicted the formation of flat pancake-like systems. This problem was solved when Bond et al. (1996) studied in detail the formation of the largescale structure. They found that primordial tidal fields play an important role in the evolution of structure, thus the dominant elements are filaments, not flat pancakes.

Bond et al. (1996) showed why the cosmic web has a filamentary character, but could not explain, why there is a large variety of systems from small groups and weak filaments to rich clusters and superclusters. It is clear intuitively that the main reason for the formation of systems of various richnesses is the presence of density perturbations of different scales. This idea is justified by comparing the HDM and CDM scenarios - the fine structure of the cosmic web is generated by density fluctuations of medium and small scales that are absent in the HDM model.

The role of very large density waves on the formation of the structure has been studied by a number of authors. Knebe et al. (2000), Bagla \& Prasad (2006), and Bagla et al. (2009) investigated the effect of the resolution of cosmological simulations on the properties of the filamentary web. Power \& Knebe (2006) studied the impact of the simulation box size on the properties 
of the simulated cosmic web. Bernardeau et al. (2002) gave a review of how non-linear perturbation theory helps us to understand the large-scale structure of the Universe and various statistical tools used to characterise its properties.

It is well known that quasi-relaxed systems of galaxies, such as groups and clusters, are formed by perturbations, which have entered the non-linear stage of their evolution. The scale of the non-linearity is usually taken equal to about $8 h^{-1} \mathrm{Mpc}$. The richest non-percolating systems of galaxies are superclusters. Rich superclusters form a cellular distribution, with large voids surrounded by rich superclusters. The characteristic diameter of these supervoids is of the order of $100 h^{-1} \mathrm{Mpc}$ (Einasto et al. 1994, 1997b).

Supervoids are not empty but contain a hierarchy of voids, as demonstrated by many authors (Martel \& Wasserman 1990; Lindner et al. 1995; Gottlöber et al. 2003; Aragón-Calvo et al. 2007; von Benda-Beckmann \& Müller 2008; van de Weygaert et al. 2009; van de Weygaert \& Platen 2009; Aragon-Calvo et al. 2010a; Jones et al. 2010). It is natural to expect that the web of rich superclusters is formed by density perturbations of scale $100 \mathrm{~h}^{-1} \mathrm{Mpc}$ and above. The skeleton of the cosmic web is discussed by, among others, Sheth \& van de Weygaert (2004), Hahn et al. (2007), Forero-Romero et al. (2009), Sousbie et al. (2008, 2009), Aragón-Calvo et al. (2010b), Bond et al. (2010b,a), and Einasto et al. (2011). Thus, we can say that the role of smallscale and large-scale density perturbations is relatively well understood. However, it remains unclear how perturbations of intermediate scale combine to form elements of the cosmic web.

We aim to investigate in greater detail than before the role of density perturbations of different scales in the formation of the cosmic web. We perform numerical simulations of the formation and evolution of the large-scale structure, using different box sizes and resolutions. Furthermore, to elucidate the influence of perturbations of different scales, a varying large-scale cutoff is introduced into the power spectrum of initial perturbations. All simulations of a given series have identical initial conditions with random initial positions and velocities of test particles, but the amplitude of all perturbations on a scale exceeding a given one is forced to be zero. In this way, we can follow how systems of galaxies grow under the influence of perturbations of various scales.

To characterise the effect of varying the cutoff scale of density perturbations, we consider three quantitative tests: the mean radii of voids defined by groups and clusters of galaxies, the mass functions of clusters of galaxies, and the density distributions of particles in the void and the supercluster core regions.

The paper is organised as follows. In the next section, we describe numerical simulations used to model the evolution of the cosmic web. For comparison, we also calculate the SDSS luminosity density field. In Sect. 3, we analyse the cluster mass functions and void sizes. We analyse the density distributions in the void and the supercluster core regions and discuss our results in Sect. 4. The last section presents the summary of the analysis.

\section{Modelling the evolution of the cosmic web}

\subsection{The SDSS luminosity density field}

To compare simulated void and cluster data with actual data, we used the recently completed Sloan Digital Sky Survey (SDSS) Data Release 7 (DR7) (see Abazajian et al. 2009). In the present analysis, we used only the contiguous northern zone of the DR7. The analysis was made in several steps. First we calculated the luminosity density field. We estimated the total luminosities of groups and isolated galaxies in a flux-limited sample, using luminosity weights that take into account galaxies and galaxy groups too faint to fall into the observational window of absolute magnitudes at the distance of the galaxy. For details of the data reduction, we refer to Tago et al. (2010) and Tempel et al. (2011). The high-resolution luminosity density field was calculated with the $B_{3}$ spline of kernel size $1 h^{-1} \mathrm{Mpc}$.

The high-resolution luminosity density field was found using spherical coordinates (the SDSS coordinates $\eta$ and $\lambda$ ) and distance. To obtain an impression of the luminosity density field of the whole northern region we show in Fig. 1 the luminosity density field in a spherical shell at a distance of $240 \mathrm{~h}^{-1} \mathrm{Mpc}$ from ourselves. It is based on the SDSS angular coordinates $\eta$ and $\lambda$, and the comoving distances $d_{\mathrm{gal}}$ of galaxies

$x=-d_{\mathrm{gal}} \lambda$,

$y=d_{\mathrm{gal}} \eta \cos \lambda$

$z=d_{\mathrm{gal}}-d_{0}$,

where $d_{0}=50 h^{-1} \mathrm{Mpc}$ is the minimal distance used in the calculation of the density field. A supercluster catalogue based on the luminosity density field of the full contiguous northern SDSS region was published by Liivamägi et al. (2010). The representation in spherical coordinates serves basically for illustrative purpose, as it is practically free of distance dependent selection effects. The spherical shell has a thickness of $10 \mathrm{~h}^{-1} \mathrm{Mpc}$ to enhance the filamentary distribution of galaxies, clusters, and superclusters. The mean distance of the shell corresponds to the distance of the Sloan Great Wall, seen in the lower area of the figure. We see that it consists of two very rich superclusters: SCL111 and SCL126 according to the catalogue by Einasto et al. (2001).

\subsection{Simulation of the cosmic web for density perturbations of various scales}

To understand the formation of the filamentary superclustervoid web correctly, we must perform numerical simulations for a box that contains both small and large waves. The smallest units of this network are galaxies, the most frequent systems of galaxies are groups and clusters of galaxies. The characteristic scale of groups is $1 \mathrm{~h}^{-1} \mathrm{Mpc}$ (galaxies are still about 10 to 100 times smaller), thus the simulation must have a resolution of at least this scale. On the other hand, the largest nonpercolating systems of galaxies are superclusters, which have a characteristic scale of $100 \mathrm{~h}^{-1}$ Mpc (Oort 1983; Zucca et al. 1993; Einasto et al. 1994; Kalinkov \& Kuneva 1995; Einasto et al. 1997a, 2001; Erdoğdu et al. 2004; Einasto et al. 2007). Superclusters have rather different richness, from small systems like the Local Supercluster to very rich systems such as the Shapley Supercluster. It is clear that this variety has its origin in density perturbations of still larger scales. Thus, to understand the supercluster-void phenomenon correctly, the influence of very large density perturbations should also be studied.

To derive both a high spatial resolution and the presence of density perturbations on a large scale interval, we used a number of simulations in boxes of sizes from $100 \mathrm{~h}^{-1} \mathrm{Mpc}$ to $768 h^{-1} \mathrm{Mpc}$, and various resolutions of $N_{\text {grid }}^{3}=256^{3}$ and $N_{\text {grid }}^{3}=512^{3}$ particles and simulation cells. To see the effect of perturbations of various scales, we use simulations with the full power spectrum, as well as with a power spectrum truncated at wave-numbers $k_{\mathrm{t}}$, so that the amplitude of the power spectrum on large scales is zero i.e., $P(k)=0$, if $k<k_{\mathrm{t}}$, wavelength $\lambda_{\mathrm{t}}=2 \pi / k_{\mathrm{t}}$. 

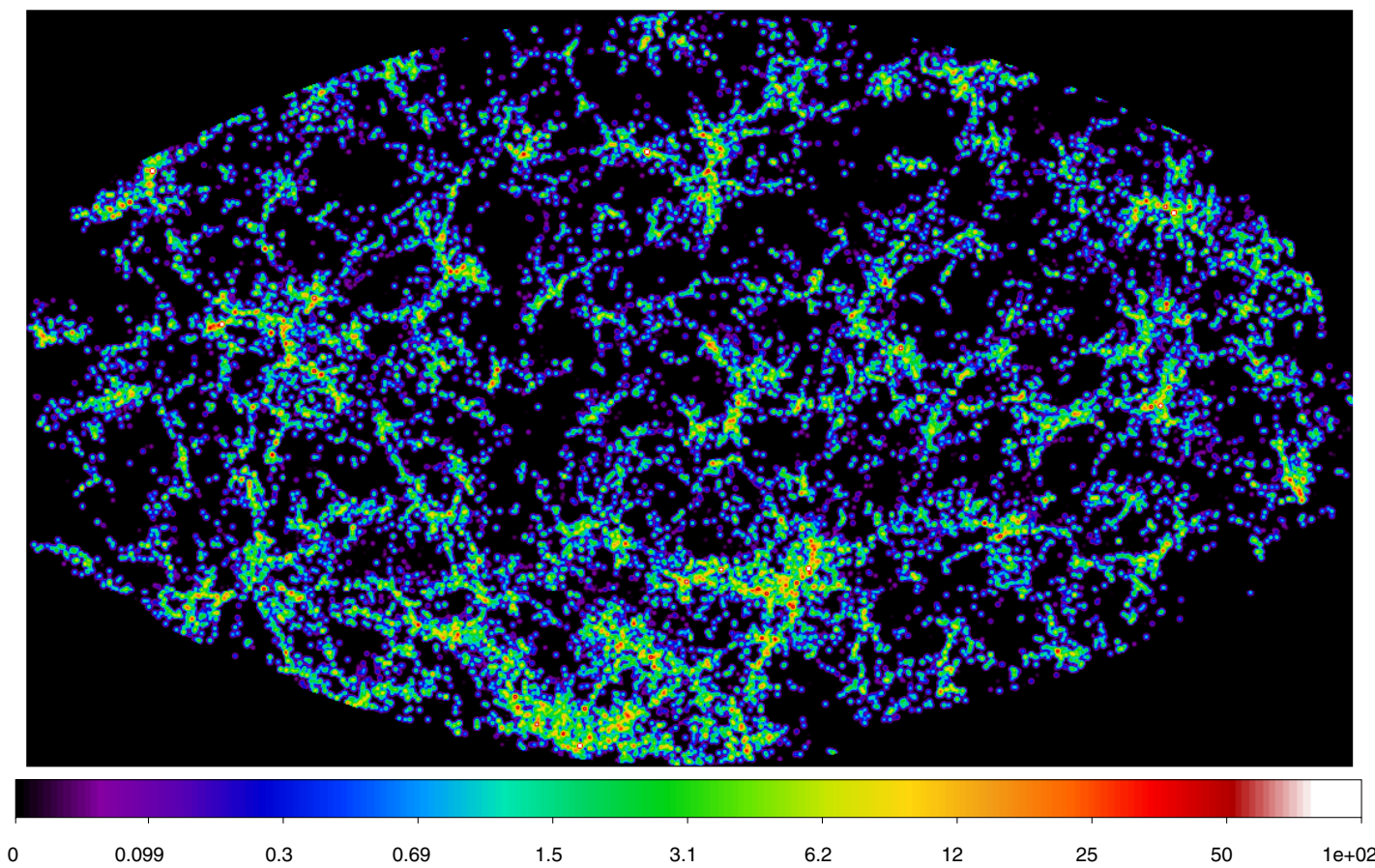

Fig. 1. The luminosity density field of the SDSS in a spherical shell of $10 h^{-1} \mathrm{Mpc}$ thickness at a distance of $240 h^{-1} \mathrm{Mpc}$. To enhance the faint filaments in voids between the superclusters, the density scale is logarithmic, in units of the mean luminosity density for the whole DR7. The rich complex in the lower area of the picture is part of the Sloan Great Wall; it consists of two very rich superclusters, SCL 111 and SCL 126 in the list by Einasto et al. (2001). The coordinates $x, y$ are defined by Eqs. (1) and (2). A cellular distribution of superclusters and filaments of various richness can be clearly seen.

All models of the same series have the same realisation of random Fourier amplitudes, so the role of different waves in the models can be easily compared. To compare different models of a series, every particle has an identification number, the same for all models of the same series. The main model parameters are given in Table 1 . In addition to models with cuts on large scales, we calculated a model, where the density perturbations on intermediate scales were truncated to zero at the wavelengths 8-64 $h^{-1} \mathrm{Mpc}$, the model M256.864. This model shows the effect of the absence of perturbations of medium scales.

We use the following notation for our models: the first characters $\mathrm{M}$ and $\mathrm{L}$ designate models with resolutions of $N_{\text {grid }}=256$ and $N_{\text {grid }}=512$, respectively; the following number gives the size of the simulation box, $L$, in $h^{-1} \mathrm{Mpc}$; the subsequent number indicates the maximum wavelength used in the simulation, also in $h^{-1} \mathrm{Mpc}$; if the full power spectrum is used these two numbers coincide. The locations of the cells inside the cubical density grid are marked by cell indices $(i, j, k)$, where $i, j$, and $k$ are integers that run from 1 to $N_{\text {grid }}$.

For the models of the M256 and the M768 series, we used in simulations the AMIGA code, which is the follow-up of the multi-level adaptive particle mesh (MLAPM) code by Knebe et al. (2001). This code uses an adaptive mesh technique in the regions where the density exceeds a fixed threshold. In this code, gravity is automatically softened adaptively, so that the softening length is close to its optimum value in both high and low-density regions. We chose a maximum level of eight refinements. For the models of the L100 and L256 series, we used the GADGET-2 code with gravitational softening length $10 h^{-1} \mathrm{kpc}$ and $20 h^{-1} \mathrm{kps}$, respectively (Springel et al. 2001; Springel 2005). The simulations M256 and M768 were performed at the Tartu Observatory, the simulation L100 at the
Table 1. Parameters of the models.

\begin{tabular}{lrrrr}
\hline \hline Model & $L$ & $\lambda_{\text {cut }}$ & $N_{\text {cl }}$ A & $N_{\text {cl }}$ B \\
& $(1)$ & $(2)$ & $(3)$ & $(4)$ \\
\hline M768.768 & 768 & 768 & 102783 & \\
M768.128 & 768 & 128 & 105496 & \\
M768.032 & 768 & 32 & 184193 & \\
M768.012 & 768 & 12 & 213569 & \\
M256.256 & 256 & 256 & 54224 & 5578 \\
M256.064 & 256 & 64 & 56000 & 6077 \\
M256.032 & 256 & 32 & 60985 & 6996 \\
M256.016 & 256 & 16 & 70813 & 7624 \\
M256.008 & 256 & 8 & 121022 & 125 \\
M256.864 & 256 & $8-64$ & & \\
L256.256 & 256 & 256 & 341758 & 59100 \\
L256.128 & 256 & 128 & 343706 & 59313 \\
L256.064 & 256 & 64 & 351198 & 61831 \\
L256.032 & 256 & 32 & 372298 & 68221 \\
L256.016 & 256 & 16 & 426699 & 84691 \\
L256.008 & 256 & 8 & 546414 & 126832 \\
L100.100 & 100 & 100 & 54348 & 173655 \\
L100.032 & 100 & 32 & 57878 & 182675 \\
L100.016 & 100 & 16 & 62396 & 197053 \\
L100.008 & 100 & 8 & 71726 & 237499 \\
\hline
\end{tabular}

Notes. Column 1: $L$ - the size of the simulation box in $h^{-1} \mathrm{Mpc}$; Col. 2: $\lambda_{\text {cut }}$ - the cut-off scale in $h^{-1} \mathrm{Mpc}$; Col. 3: the number of DF clusters in the high-resolution density field at a redshift $z=0$, for the model L256 using the parameter set A (see Sect. 2.5 for explanation); Col. 4: the number of DF clusters in the high-resolution density field at a redshift $z=0$, for the model L256 using the parameter set B; the number of AHF halos at the redshift $z=0$ for the models M256 and L100.

Astrophysical Institute Potsdam, and the simulation L256 at the High Performance Computing Centre of the University of Tartu. 
$\mathrm{M} 256256 \mathrm{z}=30$

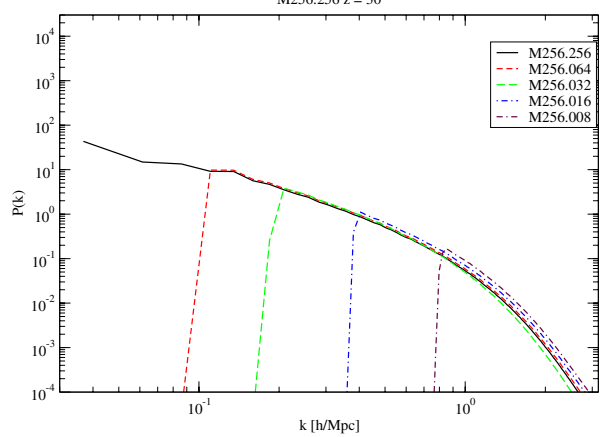

M256256z $=0$

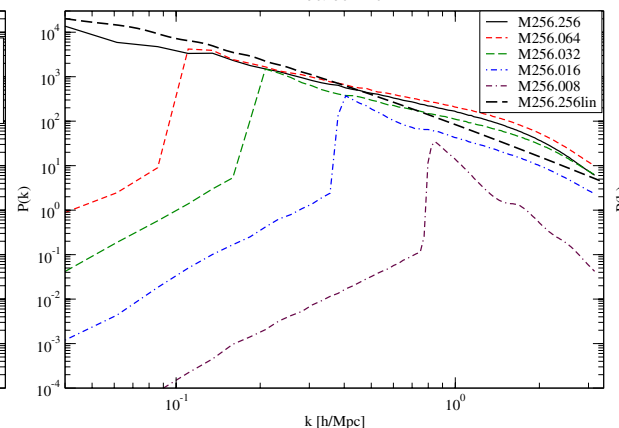

1256864

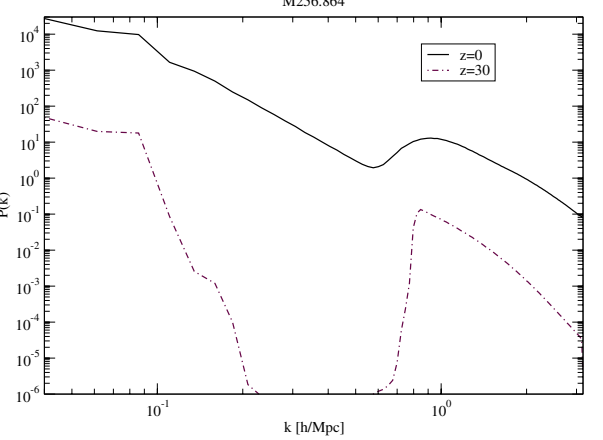

Fig. 2. The left and middle panels show the power spectra for the models of the series M 256 at the epochs $z=30$ and $z=0.00$, respectively. The right panel shows the power spectra for the model M256.864 at the epochs $z=30$ and $z=0.00$. In the middle panel, we also show the power spectrum for the full model M256.256 at the present epoch $z=0$ where the evolution has been linear on all scales.

The initial density fluctuation spectra were generated using the COSMICS code by Bertschinger $(1995)^{1}$. We assumed the cosmological parameters $\Omega_{\mathrm{m}}=0.28, \Omega_{\Lambda}=0.72, \sigma_{8}=0.84$, and the dimensionless Hubble constant $h=0.73$; to generate the initial data, we used the baryonic matter density $\Omega_{\mathrm{b}}=0.044$ (Tegmark et al. 2004). We generated initial conditions for a truncated power spectrum, using the full spectrum to calculate the model parameters. The amplitude of a spectrum was set to zero for $k<k_{t}$ during the calculation of the initial density field, keeping all simulation parameters fixed across the full set of realisations. The calculations were started for an early epoch, $z=100$. The particle positions and velocities were extracted for 12 epochs between the redshifts $z=30 \ldots 0$ (the models of the M256 and the M768 series), and for 7 epochs between the redshifts $z=100 \ldots 0$ (the models of the L100 and the L256 series).

\subsection{Calculation of the density field}

For every particle, we calculated the local density in units of the mean density, using the positions of 27 nearby particles. We also calculated the mean global density at the location of the particle. For this purpose, we first determined the density field, using a $B_{3}$ spline (see Martínez \& Saar 2002):

$B_{3}(x)=\frac{1}{12}\left[|x-2|^{3}-4|x-1|^{3}+6|x|^{3}-4|x+1|^{3}+|x+2|^{3}\right]$,

where function differs from zero only in the interval $x \in[-2,2]$. The one-dimensional $B_{3}$ box spline kernel of width $h=N$ is

$K_{B}^{(1)}(x ; N)=B_{3}(x / N) / N$.

This kernel preserves the interpolation property (mass conservation) for all kernel widths that are integer multiples of the grid step, $h=N$. The 3D $K_{B}^{(3)}$ box spline kernel is given by the direct product of three one-dimensional kernels

$\left.K_{B}(\boldsymbol{x} ; N) \equiv K_{B}^{(3)}(\boldsymbol{x} ; N)=K_{B}^{(1)}\right)(x ; N) K_{B}^{(1)}(y ; N) K_{B}^{(1)}(z ; N)$,

where $\boldsymbol{x} \equiv\{x, y, z\}$.

To calculate the high-resolution density field, we use the kernel of the scale equal to the cell size of the simulation, $h=L / N_{\text {grid }}$, where $L$ is the size of the simulation box, and $N_{\text {grid }}$ is the number of grid elements in one coordinate. We use consecutive smoothing stages; the smoothing stage of the order $i$

${ }^{1}$ http://arcturus.mit.edu/cosmics has the smoothing scale $r_{i}=L / N_{\text {grid }} \times 2^{i}$. The effective scale of smoothing is equal to $2 \times r_{i}$. We applied this smoothing up to index (order) 6 . The smoothing with the $B_{3}$ spline kernel is rather close to the smoothing with an Epanechnikov kernel of the same scale, used in earlier studies of superclusters (Einasto et al. 2006, 2007). For the model L100, the smoothing index 5 corresponds to the kernel of the radius $6.25 h^{-1} \mathrm{Mpc}$, for the model L256 the smoothing index 4 corresponds to the kernel of the radius $8 h^{-1} \mathrm{Mpc}$, and for the model M768 the smoothing index 2 corresponds to the kernel of the radius $12 h^{-1} \mathrm{Mpc}$. These kernel radii were used to calculate the global density field used in the selection of the void and supercluster core regions.

The power spectra of the models of the series M256 are shown in Fig. 2 for both an early epoch, $z=30$, and the present epoch $z=0.0$. The power spectra for the model M256.864 are shown in the right panel of the same figure for the same epochs. For comparison, we also show in the middle panel the power spectrum of the model M256.256 for the present epoch, if evolved linearly at all scales. Figure 2 shows that at an early epoch and at small wavelengths all spectra practically coincide. At the present epoch, the amplitudes of spectra for different models differ at small wavelengths: the models with the truncated spectra evolve at small wavelengths more slowly than the full model. At small wavelengths, the slowest evolution is seen in the model M256.008, where the growth of the amplitudes is almost linear on all scales. This effect is expected because density waves of larger size that could amplify the growth of smallscale waves are absent (see the analysis below). We also see that in all models with a cutoff, the amplitude of the spectrum in the cutoff range slowly increases, i.e. some power is translated from smaller to larger waves. This effect is much stronger in the model M256.864, where in the cutoff range of the spectrum a fairly high amplitude of the spectrum develops for the present epoch, only a factor of ten lower than the amplitude at the cutoff wavelength $\lambda=8 h^{-1} \mathrm{Mpc}$.

The high-resolution density fields at the present epoch for models of the M256 series are shown in Fig. 3.

\subsection{Comparison of halo finders}

In simulations, instead of groups and clusters of galaxies, dark matter halos are usually used to define simulated compact systems. In the pilot phase of this study, we used the PM code in a box of size $L=128 h^{-1}$ Mpc with $128^{3}$ particles and simulation cells. For these models, we applied the conventional halo finder using the FoF technique with the linking length parameter 0.2 in units of the mean separation of particles. The analysis showed 

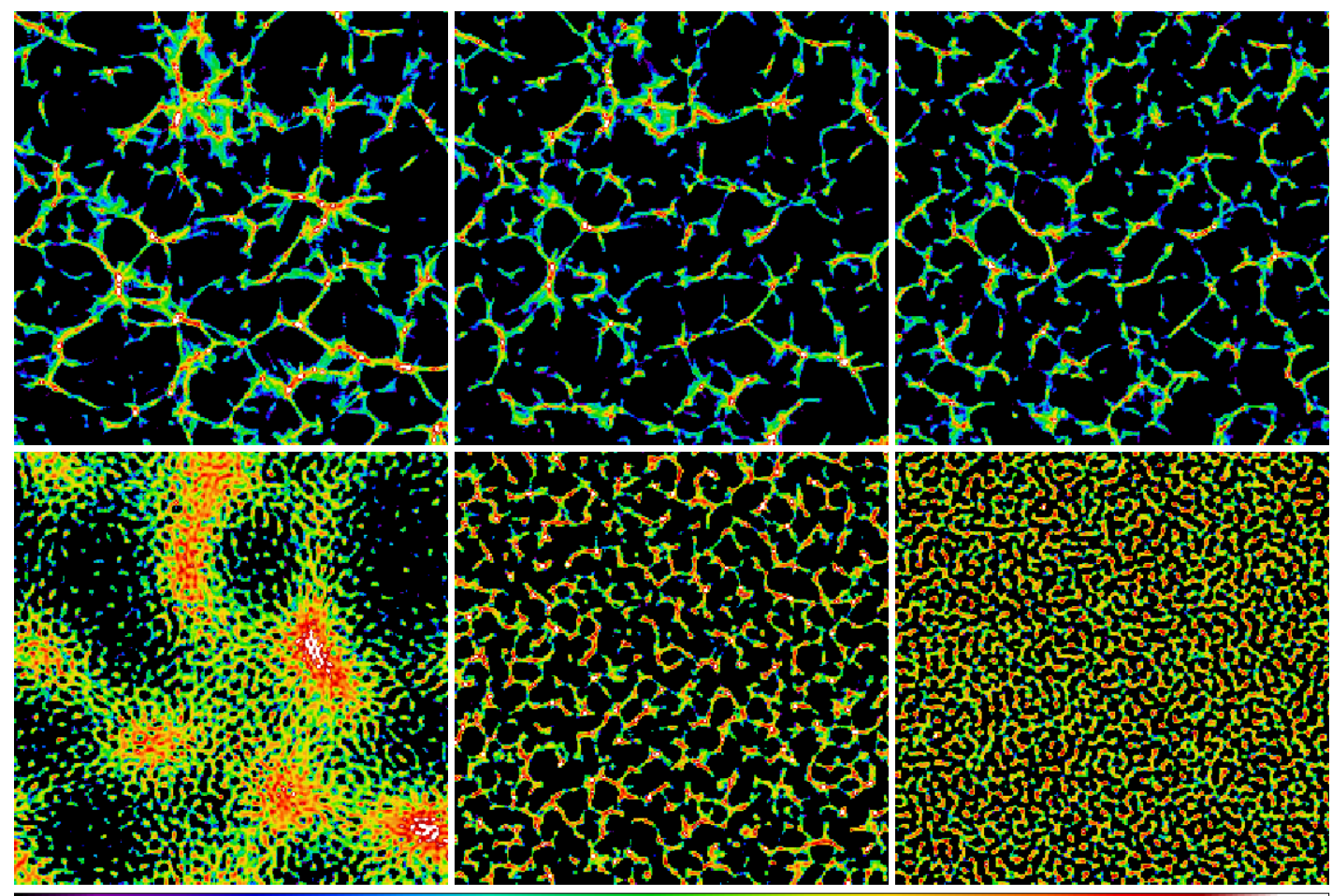

1.04

1.12

1.27

1.58

2.2

3.43

5.87

10.8

20.5

40

Fig. 3. Density fields for the models of the M256 series. The upper panels show the high-resolution fields for the models M256.256, M256.064, and M256.032, the lower panels - for the models M256.864, M256.016, and M256.008 (from left to right). The densities are shown for a layer of $6 h^{-1} \mathrm{Mpc}$ thickness at the $k=75$ coordinate. All fields correspond to the present epoch $z=0$. The densities are expressed on a logarithmic scale to enhance the low-density regions. Only the overdensity regions are shown. The upper limits to the density are 40 in the upper panels, and 10,10 , and 5 in the lower panels.

that the FoF halos are of little use for our study, since in the central regions of superclusters, the richest FoF halos contain the whole core of the supercluster, and have sizes of the order of $10 h^{-1} \mathrm{Mpc}$.

For this reason, we tried the adaptive Amiga halo finder (AHF) code developed by Knollmann \& Knebe (2009), where the number of particles in a halo is $N_{\mathrm{p}} \geq 20$. Halos and their parameters (masses, virial radii, positions, velocities etc.) were found for most models and simulation epochs. Here we have another difficulty: the number of halos for early epochs (high redshifts) decreases dramatically with increasing $z$ : there are about 1000 times fewer halos at the redshift $z=10$ in the model L100 than at the present epoch. For models of the M256 series, the AHF finds no halos at the redshifts $z=5$, and 10, about 10 times fewer halos than the density field cluster method (see below) for the models M256.256, M256.064, M256.032, and M256.016, and almost no halos for the model M256.008 (see Table 1). The density enhancements in these models are much smaller and do not conform to the definition of the AHF halos, (which have at least 20 particles), in spite these models containing many small compact density enhancements at all redshifts. In Fig. 3, we present the density fields of the models of the M256 series at the redshift $z=0$, and Figs. 1-3 of Einasto et al. (2011) for higher redshifts.

Taking these difficulties into account, we used the highresolution density field to define compact systems, i.e., density field (DF) clusters. DF clusters correspond to groups and clusters of galaxies in the real Universe, and to halos in simulations. In defining density field clusters, we applied the conventional tradition, used by Abell in his rich cluster catalogues
(Abell 1958; Abell et al. 1989). The Abell clusters were selected using galaxies in a sphere of a radius of about $1.5 \mathrm{~h}^{-1} \mathrm{Mpc}$. Our density field method to find halos is rather similar to the DENMAX method by Bertschinger \& Gelb (1991) and Gelb \& Bertschinger (1994). The basic difference lies in the detail: we do not use particles to define the center and the mass of the halo, but only data obtained directly from the density field. The procedure for finding the DF clusters is described in the next subsection.

To compare our DF cluster mass distributions with the mass distributions of the AHF halos, we applied both methods for the highest resolution model L100. We found the DF clusters using the parameters $D_{0}=2$ (in units of the mean density), $D_{\mathrm{p}}=20$ (the minimal number of particles), and $N=8$; the latter corresponds to the virial (outer) radius of the halo $R_{\text {vir }}=1.66 h^{-1} \mathrm{Mpc}$, which is similar to the virial radii of the largest halos found with the AHF method. The cumulative mass functions for the DF clusters and the AHF halos for all models of the L100 series for the redshift $z=0$ are shown in Fig. 4

Figure 4 shows that the maximum masses of halos for both halo finders are practically identical for all models with different cutoffs. The shapes of the cumulative mass functions are slightly different. Table 1 and Fig. 4 show that the total numbers of halos found with the AHF method are about three times larger than the numbers of the DF clusters for models with the same cutoff. This difference is expected, since AHF treats all subhalos as independent halos.

In the mean mass range, the number of the DF clusters is a bit higher than the number of the AHF halos. This difference is also expected. Our DF cluster finder always has an identical 

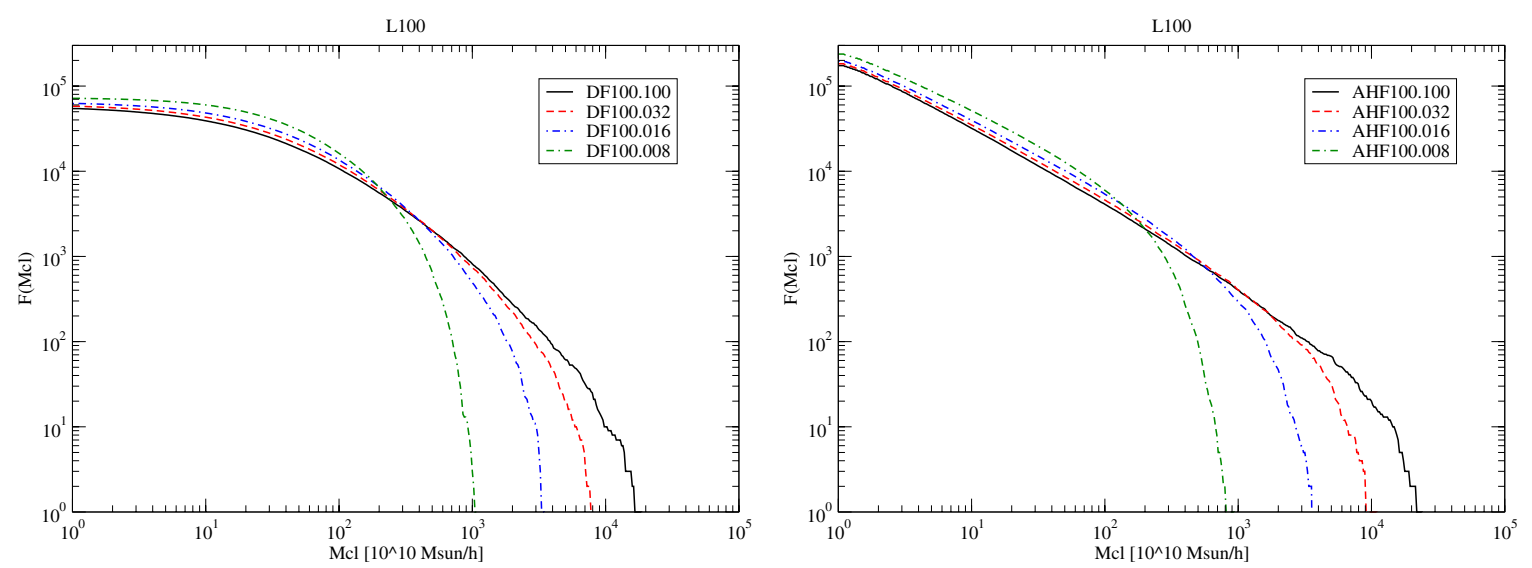

Fig. 4. The left panel shows the cumulative mass functions of the density field clusters for the model L100 for various cutoff scales. The right panel shows the cumulative mass functions of the AHF halos for the same model.

volume around a high-density peak, whereas the virial radii of the AHF halos decrease for the model L100.100 from $R_{\text {vir }}=$ $1.5 h^{-1} \mathrm{Mpc}$ to $R_{\mathrm{vir}}=0.05 h^{-1} \mathrm{Mpc}$ for the considered mass range. For this reason, close intermediate-mass halos are counted as separate small halos in the AHF method, but as a single larger halo in the DF cluster method.

These differences in the mass distributions are not important for the present study, since we compare mass distributions for different cutoffs of the power spectra, obtained with the same halo identification method. We use halos basically to identify voids and to measure their radii; the internal structures of the halos are not important for the present analysis.

\subsection{Density field clusters}

As a first step, we find local density maxima of the highresolution density field. In the search for maxima, we use three parameters: the minimum density threshold $D_{0}$, above which we search for density maxima, and the minimum mass of the DF clusters, $D_{\mathrm{p}}$. During the search, densities are expressed in units of the mean density of the simulation, and masses - in units of the particle mass of the simulation (the number of particles in a halo). The mass of the DF clusters, $M_{\mathrm{cl}}$, is calculated by adding the local densities in the cells within $\pm N$ cells from the central one, i.e. in a total in $(2 N+1)^{3}$ cells. The number $N$ defines the volume where we count the mass of the cluster, and it is the third parameter of the search. We express the DF cluster masses in solar units, using the known particle mass.

For the M256 model, we used the parameters $D_{0}=2$, $D_{\mathrm{p}}=5$, and $N=3$; for the M768 model, we used the parameter set $D_{0}=2, D_{\mathrm{p}}=5$, and $N=1$, which correspond to the cluster search radii $r=3.5 h^{-1} \mathrm{Mpc}$ and $r=4.5 h^{-1} \mathrm{Mpc}$, respectively. To find the dependence of the DF cluster mass functions and the cluster-defined void radii on the parameters used in the definition of a DF cluster, we used two sets of parameters for the models of the L256 series - a high-sensitivity set A with $D_{0}=2, D_{\mathrm{p}}=5$, and $N=3$, and a low-sensitivity set B with $D_{0}=10, D_{\mathrm{p}}=50$, and $N=5$. The cluster search radii are $r=1.75 h^{-1} \mathrm{Mpc}$, and $r=2.75 h^{-1} \mathrm{Mpc}$, for the parameter sets $\mathrm{A}$ and $\mathrm{B}$, respectively. The parameter set $\mathrm{A}$ finds the DF clusters in faint filaments crossing supercluster-defined large voids, the parameter set B avoids most of the DF clusters in these filaments.

The total numbers of the DF clusters found for the present epoch $z=0$ and the selection parameters used are given in
Table 1. For the models M256 and L100, we also give the numbers of the AHF halos.

\section{Analysis of models}

\subsection{The density field of truncated models}

To have an idea of the appearance of the density fields, we show in Fig. 3 the high-resolution density fields for all the models of the series M256: M256.256, M256.064, M256.032, M256.016, M256.008, and M256.864. To emphasise the filaments joining the DF clusters, we express densities in logarithmic scale, and use sheets of a thickness $6 h^{-1} \mathrm{Mpc}$. The fields are shown for the present epoch $z=0.0$. The models M768 and L256 look similar, when cuts at the same scales are used, but fewer details are seen in the model M768 with respect to the model L256.

Figure 3 shows that the models M256.256 and M256.064 display rather similar patterns of the cosmic web. An even closer similarity is observed when we compare the density fields of the models L256.256 and L256.128. This similarity of the patterns of the cosmic web in these models shows that the pattern of the web is defined by density perturbations of scales smaller than $\simeq 100 h^{-1} \mathrm{Mpc}$.

In models with shorter scale power spectrum cuts largescale features disappear. In the models with a cutoff scale $\leq 100 h^{-1} \mathrm{Mpc}$, the shape of the pattern of the cosmic web is determined by the maximum scale of density perturbations. This is one of our main qualitative conclusions: in the absence of large-scale perturbations, galaxy systems larger than the cutoff scale do not form. In other words, the scale of the cosmic web is determined by the density waves of the largest scale present. However, this conclusion is correct only up to the scales $\sim 100 h^{-1}$ Mpc. Figure 3 (and similar plots for other models) shows that the addition of perturbations larger than this scale does not create larger systems, but only amplifies them. We study this phenomenon below.

\subsection{The role of the density waves of medium scales}

To see the effect of the absence of density perturbations of medium scales, we performed one simulation, where the amplitude of the initial density fluctuations between wavelengths $8 h^{-1} \mathrm{Mpc}$ and $64 h^{-1} \mathrm{Mpc}$ was truncated to zero. This model (M256.864) is otherwise identical to the model M256.256 and was generated using identical Fourier amplitudes. The 

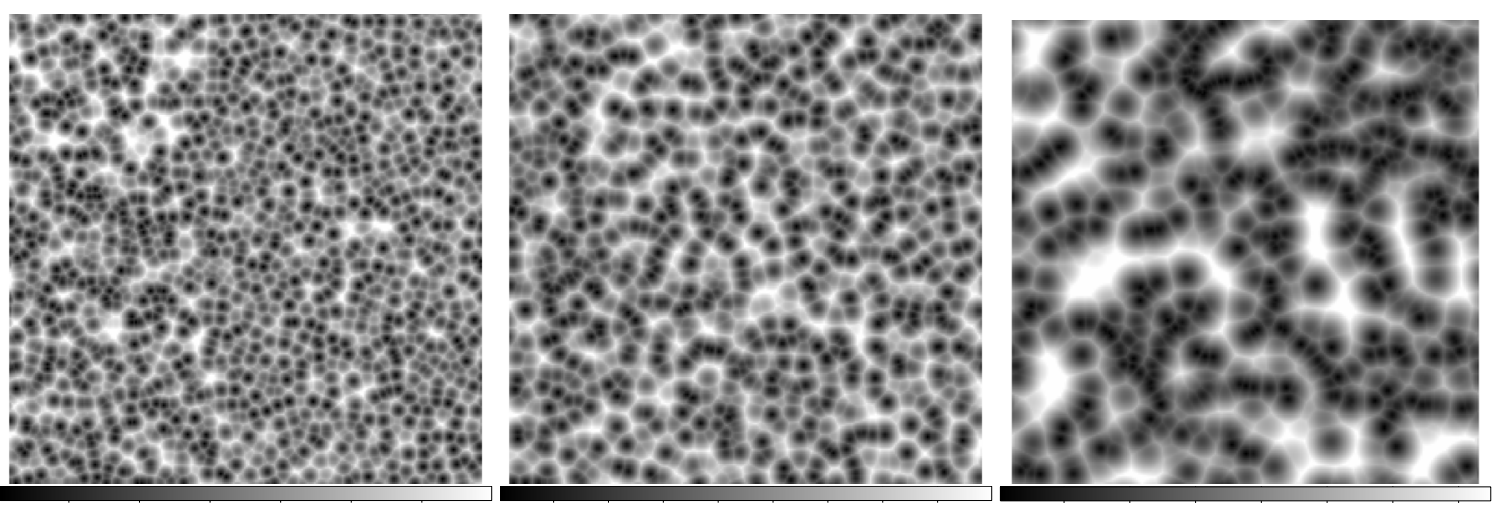

Fig. 5. The matrix of distances to the nearest DF clusters for the models M256.008, M256.016, and M256.256 (from left to right). The "density" is defined as the distance to the nearest DF cluster. The matrix is given for the $k=220 h^{-1} \mathrm{Mpc}$ coordinate. Note that changes of the mean radii in the distance matrix are similar to changes of the overdensity pattern of the density field, shown in Fig. 3.

power spectra of this model at $z=30$ and $z=0$ are shown in Fig. 2, and the density field at the present epoch in Fig. 3.

Figure 3 demonstrates very clearly the role of density perturbations of various scales in the formation of the cosmic web. In the absence of large-scale perturbations, systems larger than the cutoff scale do not form. The result of removing only the medium-scale perturbations is of particular interest. As seen from Fig. 2, during the evolution the power spectrum on intermediate scales, which is absent at the initial epoch, is in this case almost restored. However, this increase in the power spectrum amplitudes does not lead to the formation of medium-scale galaxy systems such as filaments. In a model without the initial medium-scale perturbations, filaments are absent, both within superclusters and between them. The distribution of small-scale systems is more or less random, and there are no compact systems of galaxies such as clusters - the compact systems are rather small.

\subsection{The distribution of void sizes}

Cosmic voids are regions of space devoid of certain kinds of objects - galaxies, clusters of galaxies etc. Different types of objects define voids of different size. The reason for the dependence of void sizes on the mass (or the luminosity) of objects used in their definition is simple. Large voids are determined by rich clusters and crossed by filaments of faint galaxies. Moreover, almost all systems of galaxies contain outlying faint members (see Fig. 1 for the luminosity density field of a spherical shell of the Sloan Digital Sky Survey). Dwarf galaxies define much smaller voids than giant ones (Einasto et al. 1986, 1989, 1991; Lindner et al. 1995, 1996; Gottlöber et al. 2003; von Benda-Beckmann \& Müller 2008). For the hierarchy of voids, we also refer to van de Weygaert \& van Kampen (1993), Peebles (2001), Gottlöber et al. (2003), Aragón-Calvo et al. (2007), van de Weygaert \& Platen (2009), Aragon-Calvo et al. (2010a), and Aragón-Calvo et al. (2010b).

To find the distribution of void radii, we used a simple void finder suggested by Einasto et al. (1989). For comparison of different void finders, we refer to Colberg et al. (2008). For each vertex of the simulation grid, we first calculated its distance to the nearest DF cluster. For the positions of the DF clusters, we used the $i, j, k$-indices of the maximum local density cells. The distance matrix is similar to the density field matrix; for the models M256.008, M256.016, and M256.256, these matrices are shown in Fig. 5.
Figure 5 shows the maxima of the distance matrix field. These maxima correspond to the centres of voids, and their values are the void radii. The distribution of clusters is noisy, thus there are many nearby local maxima in the distance matrix field. We define the position of the void centre as the location of the cell, which has the highest distance value within a box of the size of \pm 3 grid elements.

Figure 5 shows that in some places there are long ridges in the distance matrix; these ridges delineate elongated voids. We consider them to be individual voids, if their centres are separated by more than $3 h^{-1} \mathrm{Mpc}$, i.e. they count as separate entries in the void search using the criterion shown above.

In models with a higher cutoff, the maximum void radii increase with the cutoff scale, but only moderately. There is only a small difference in the distribution of void radii between the models M256.256 and M256.064. In other words, very large density waves do not change the structure of the superclustervoid network, their role consisting essentially of the amplification of existing structures.

The mean void radii were found for a broad range of the DF cluster mass thresholds, $M_{\text {th }}$. The highest threshold used was selected so that the volume density of the DF clusters in the sample is approximately equal to the volume density of the Abell clusters, at about $25 \times 10^{-6}\left(h^{-1} \mathrm{Mpc}\right)^{3}$ (Einasto et al. 2006). This gives $\simeq 420 \mathrm{DF}$ clusters in the volume of the L256 model. The actual number of DF clusters used in the void definition depends on the value of the DF cluster mass threshold, $M_{\mathrm{th}}$, used in the void search.

In Fig. 6, we show the mean void radii as functions of the threshold mass $M_{\mathrm{th}}$ of the DF clusters used in void search. This figure shows that the void radii depend on the threshold mass, as expected from similar studies of the void radii in galaxy samples of different threshold luminosity, demonstrated in many studies cited above. We study this effect in more detail below.

\subsection{The distribution of the DF cluster masses}

One of the characteristics of the cosmic web is the distribution of cluster masses. The cumulative mass functions of the DF clusters for the models of the series L256 and M768 are shown in Fig. 6. We see that DF cluster masses strongly depend on the scale of the power spectrum cutoff. All models with a cutoff on the scale $8 h^{-1}$ Mpc have DF cluster mass distributions with rather sharp decreases on the high mass side. The maximum masses 

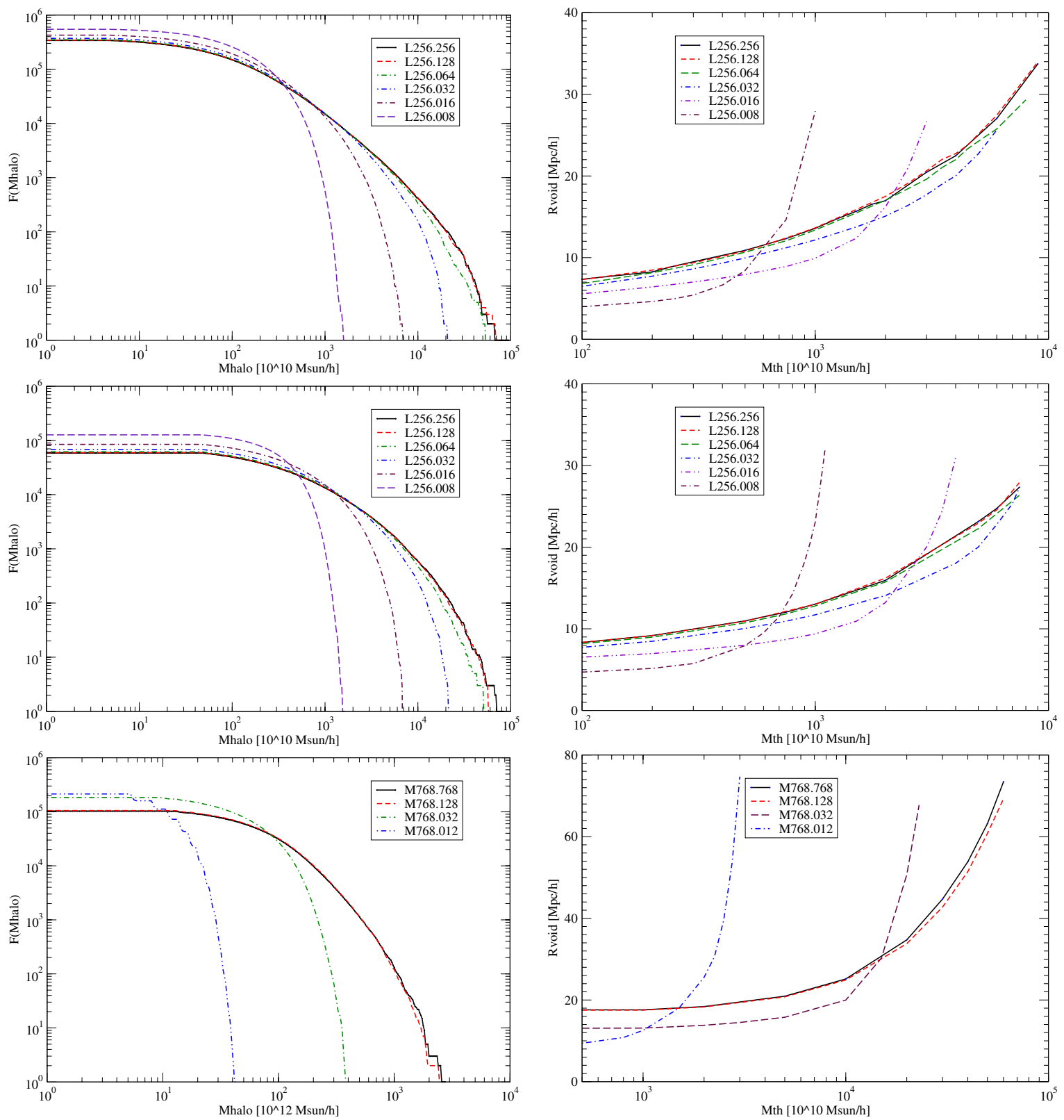

Fig. 6. The left panels show the cumulative mass functions of the density field clusters for the models with various cutoff scales. The right panels show the mean radii of voids, defined by the DF clusters for different threshold masses, $M_{\mathrm{th}}$, and for various cut scales. The upper panels are for the model L256 with the DF cluster search parameter set A, the middle panels are for the same model with the DF cluster search parameter set B, the lower panels are for the model M768.

of the DF clusters in these models are $\simeq 1.5 \times 10^{13} M_{\odot}$ for the L256 model and $\simeq 4 \times 10^{13} M_{\odot}$ for the M768 model. The maximum masses of the DF clusters in the models of the L100 series are lower than the maximum masses of the DF clusters in the models of the L256 series. These differences are due to the larger sizes of the boxes used in the DF cluster search in the models of the L256 and the M768 series. As shown in Fig. 4, there are practically no differences between the maximum masses of the DF clusters and those the AHF halos of the L100 model, since in the DF cluster search we used a search radius approximately equal to the virial radius of the most massive AHF halo.

With increasing spectrum cutoff scale, the mass distributions rapidly shift to higher masses. This rapid increase in the maximum DF cluster mass continues up to the cutoff scale of $64 h^{-1} \mathrm{Mpc}$. A higher cutoff scale for the power spectrum only moderately increases the maximum masses of the DF clusters. This increase almost stops at the cutoff scale $128 h^{-1} \mathrm{Mpc}$. The most massive DF clusters in these models have the masses $\simeq 7 \times$ $10^{14} M_{\odot}$ and $\simeq 2 \times 10^{15} M_{\odot}$, for the models L256 and M768, respectively.

The number of low mass DF clusters in the models depends on the selection parameters used in the cluster search. The total number of DF clusters in the model L256 found for the highsensitive and the low-sensitive parameter sets, A and B, is rather different. The high-sensitive set A with a lower search peak density level $D_{\mathrm{p}}=5$ has about six times more DF clusters than the set B with $D_{\mathrm{p}}=50$. All additional DF clusters found for the parameter set $\mathrm{A}$ are in the low mass range, as seen in Table 1 and Fig. 6. In the higher mass region, the cumulative mass functions for both sets of search parameters almost coincide. As we see below, in the void search both parameter sets yield voids of approximately equal mean radii for identical threshold masses. This shows that our results are rather robust to the parameter choice in the DF cluster definition. 
A more detailed study shows, however, that density waves of still larger scales influence the structure of the cosmic web. We discuss below the evolution of the density field in the void and supercluster core regions.

\section{Discussion}

\subsection{Voids defined by clusters of different mass}

As seen in Table 1 and Fig. 6, the numbers of the DF clusters for the search parameter set A for the models L256 are about six times larger than for the parameter set $\mathrm{B}$. The basic reason for this difference is the use of a much lower threshold density $D_{0}=2$ and the peak density $D_{\mathrm{p}}=5$ in the cluster search; for the set $\mathrm{B}$, the corresponding densities are $D_{0}=10, D_{\mathrm{p}}=50$. For instance, the number of the DF clusters in the L256.256 model for the lowest mass threshold $10^{12} M_{\odot}$ is 149265 for the parameter set A, and 59100 for set B. In spite of the large difference in the number of the DF clusters for different search parameters, the mean void radii for both parameter sets are approximately equal, as seen in the right panels of Fig. 6. A detailed inspection of the density field maps shows that this similarity in void sizes has a simple reason: the DF clusters of the set B are high-density peaks inside the same filaments that form the basic web of more densely populated filaments of the DF clusters of the set A. The density field maps also show that there are no extra faint clusters inside the relatively small voids defined by the clusters of the set A. For void definition, it is irrelevant how densely populated the filaments surrounding the voids are, if there are no galaxies outside the filaments.

This conclusion agrees with the results of earlier studies by Lindner et al. (1996), Peebles (2001), and Gottlöber et al. (2003) among others, that voids defined by relatively faint galaxy filaments are completely devoid of galaxies.

Figure 6 shows that for a low DF cluster mass threshold, the void radii are almost independent of the mass threshold $M_{\mathrm{th}}$. This means that in this DF cluster mass interval the clusters are located in identical filaments.

If the DF cluster mass threshold increases further, the void radii start to increase. This means that some filaments are fainter than the respective mass threshold limit, and do not contribute to the void definition. This slow increase in the mean void radii $R_{\mathrm{v}}$ as a function of the DF cluster mass threshold $M_{\mathrm{th}}$ for strongly cut models is valid until a certain $M_{\text {th }}$ value, of about $3 \times 10^{12} M_{\odot}$ and $10 \times 10^{12} M_{\odot}$ for the L256.008 and the L256.016 models, respectively. Thereafter, with an increasing mass threshold, the void radii increase very rapidly until clusters disappear. This effect is due to the very sharp decrease in the number of DF clusters of a high mass (see the left panels of Fig. 6). These rare clusters define very large voids. The sizes of these voids are not characteristic of the overall cosmic web pattern of the particular model.

Figure 6 shows that in the models with a higher cutoff scale $\lambda_{\text {cut }} \geq 32 h^{-1} \mathrm{Mpc}$, the void mean radii grow with the growth of the mass threshold smoothly, until the highest masses, which correspond to very rich clusters. Moreover, the $R_{\mathrm{v}}$ versus $M_{\text {th }}$ curves for the models M256.032, M256.064, M256.128, and M256.256 are almost identical. This means that the scale of the cosmic web is determined essentially by density perturbations of a scale up to $32 h^{-1} \mathrm{Mpc}$. Some differences in the $R_{\mathrm{v}}$ versus $M_{\text {th }}$ curves remain between the models M256.032 and M256.064. The higher cutoff models are practically identical in this void size test.
Thus, the void analysis confirms our results from the mass distribution of the DF clusters, that density perturbations of large scales have little effect on the pattern of the cosmic web as characterised by void sizes.

The largest voids in the L256.256 model are defined by the rich clusters of mass threshold $9 \times 10^{13} M_{\odot}$, and have the mean radii $\simeq 30 h^{-1} \mathrm{Mpc}$. These voids are characteristic of filaments, which divide supercluster-defined voids (supervoids) into approximately equal sub-voids.

The model M768 has a lower resolution; here faint filaments are absent, and the scale of voids is defined by the DF clusters of higher mass. As in the model L256, models with a smaller cutoff scale have smaller voids. The mean void radii for the models M768.128 and M768.768 have practically identical $R_{\mathrm{v}}$ versus $M_{\text {th }}$ curves. The largest voids in this model are defined by the DF clusters of the mass threshold $6 \times 10^{14} M_{\odot}$. These very rich clusters populate the cores of rich superclusters, thus the respective void radii are practically equal to the radii of supervoids, $R_{\mathrm{v}} \simeq 70 h^{-1} \mathrm{Mpc}$ (Lindner et al. 1995; Einasto et al. 1997a).

\subsection{The evolution of the density field in the void and supercluster core regions}

As an additional test, we studied the evolution of the density field in extreme voids and supercluster cores. Here we used the property of our models - all variants of a series with a different spectrum cutoff scale were generated with an identical random number set. Thus we can use the particle ID numbers to study the behaviour of the web on different spectrum cutoff scales.

To define the extreme void and supercluster core regions we used the global density field smoothed with a wider kernel, applying the same procedure as explained above for finding the DF clusters in the high-resolution density field. To calculate the global density field, we used for the models of the series L256 the kernel size $8 h^{-1} \mathrm{Mpc}$, for the models of the series M768 the kernel size $12 h^{-1} \mathrm{Mpc}$ when applying the $B_{3}$ spline. For every particle, we store the local density value at the particle location (found on the basis of 27 nearest neighbours) in addition to its coordinates, and the global density found from the density field with a large smoothing length as described above. These data are available for all models and evolution steps. We also found the distributions of the local and global density of particles.

Using this information, we extracted particle samples for models with the full power spectra at the present epoch, which had $\simeq 5 \%$ of particles with the lowest and the highest global density values. We refer to these particle samples as the void and core samples, respectively. The number of particles in the void and core samples of the M768.768 and the L256.256 models are given in Table 2. We calculated the cumulative distributions of the local densities of the void and core particles at the present epoch $z=0$ for all the models of the L256 and M768 series. The results are shown in Fig. 7.

We discuss first the cumulative density distribution of the void and core particle samples of the M768 model. This model has a lower resolution and lacks the low-mass DF clusters in the deep void regions, so the interpretation of the results is simpler. Figure 7 shows that the cumulative particle density distributions of the void and core regions of the model M768.012 are almost identical. Both distributions are rather symmetrical around the mean density level $D_{\text {loc }}=1$. This result shows that in this model, there is no difference between the void and core regions. The symmetry around the mean density level indicates that the evolution is still close to the linear regime of the growth of density perturbations, i.e. the growth of the negative and positive 

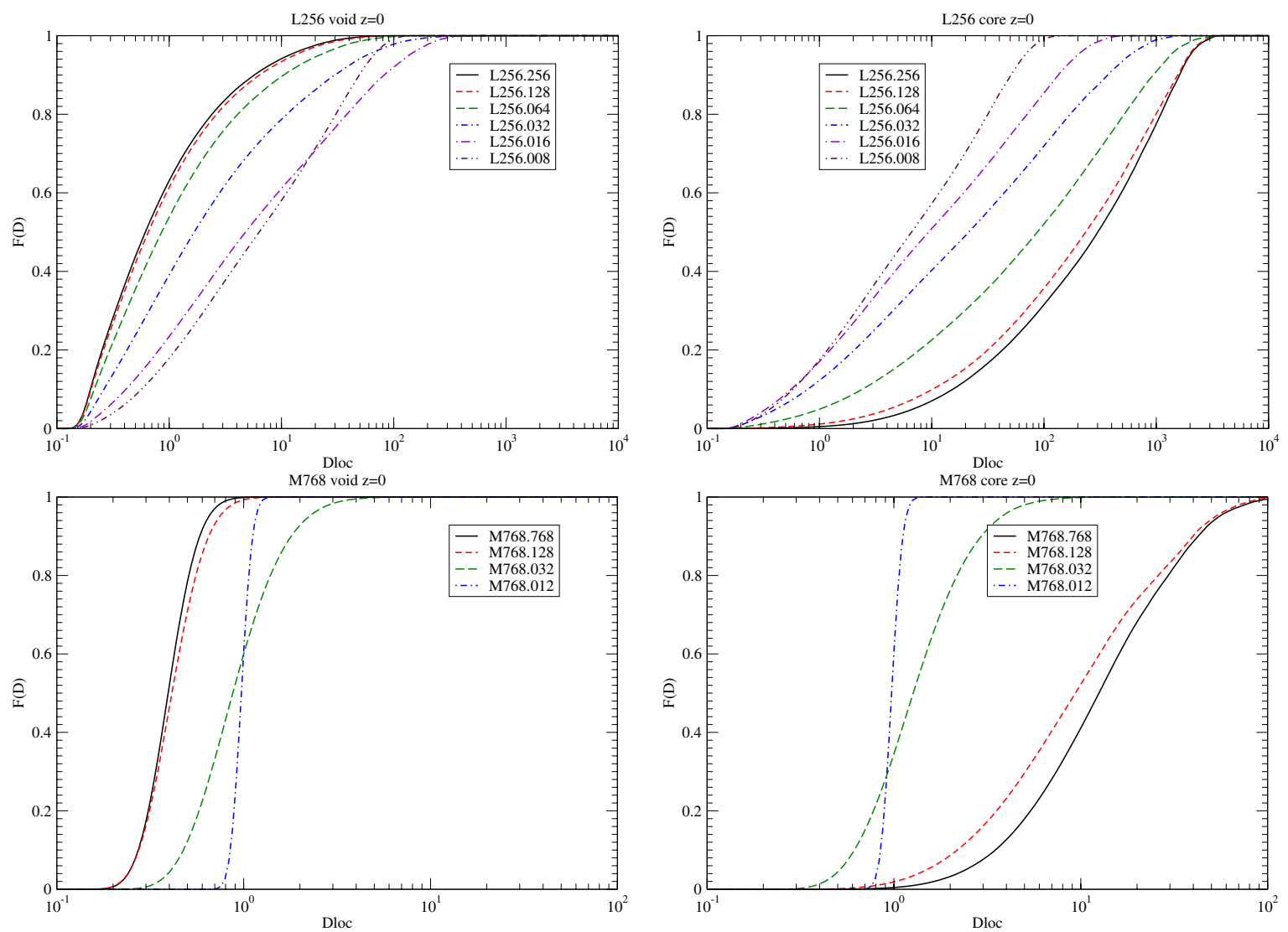

Fig. 7. The cumulative distributions of the local densities for particles in the void and core regions for the present epoch $z=0$ are given in the left and the right panels, respectively. The upper row is for the L256 models, the lower row for the M768 models. Models with various cutoff scale of the power spectra are shown.

Table 2. Results of the K-S test.

\begin{tabular}{lrrr}
\hline \hline Model & $n$ & $d$ & $p$ \\
\hline M768 void & 820002 & 0.07563 & 0.000000 \\
M768 core & 823717 & 0.12304 & 0.000000 \\
L256 void & 6677929 & 0.01747 & 0.000000 \\
L256 core & 6768219 & 0.04725 & 0.000000 \\
\hline
\end{tabular}

Notes. The meaning of $n, d$ and $p$ is explained in text.

sections of the density contrast $\delta=D-1$ is similar and proportional to the growth factor of the evolution.

In the model M768.032, the amplification of the density growth by the density waves of medium scale is already visible. The difference between the density distributions of the void and core samples is still small - there are no big voids and no rich supercluster cores.

The distributions of the particle densities in the void and core regions are completely different in the models M768.128 and M768.768. In the void regions, all local densities are lower than the mean density, i.e. there are no systems of galaxies. We recall that for the formation of a galaxy or a galaxy group the local density must exceed a certain threshold, about 1.6 in the mean density units. In contrast, in the core regions there are almost no particles of local densities less than the mean density.

We also note that there is a small but definitely significant difference between the distribution of densities in the void regions of the models M768.128 and M768.768, and in the core regions of the same models. The difference is smaller in the void regions, but in both regions it is clearly present. The results of the Kolmogorov-Smirnoff test of the comparison of the cumulative distributions of particle densities in the void (core) regions of the M768.128 versus M768.768 and the L256.256 versus L256.128 models are shown in Table 2, where $n$ is the number of particles in respective samples (which are identical in the full model and the $128 h^{-1}$ Mpc cut models of the same series), $d$ is the maximum difference of cumulative distributions, and $p$ is the probability that the distributions compared are taken from the same parent sample. As we see, this test shows that the distributions are different at high significance levels. This shows that the density perturbations of a larger scale than $128 h^{-1}$ Mpc make voids emptier and systems of galaxies richer, i.e. they amplify the emptiness and the richness of the cosmic web.

The model L256 has much higher spatial and mass resolutions. For this reason, there are more differences between the particle local density distributions of the void and core regions. In this model in the void regions we see the presence of particle overdensities, with local densities $D>1$. These particles form faint filaments crossing large voids. It is important that the larger the power spectrum cutoff scale (i.e. the larger perturbations are included in the simulation), the lower the fraction of the number of particles with overdensities. In other words, large-scale perturbations suppress the formation of filaments in void regions.

In the core regions the growth of overdensities is more rapid, the larger is the scale of the perturbations included in simulations. In the model L256.256, there are no particles with local densities below the mean density, i.e. all particles have been moved to rich systems. Owing to the higher resolution, the highest densities in the core regions are much higher than in the model M768. 


\subsection{Why the perturbations of the largest scales do not increase the scale of the cosmic web?}

Our study suggests that density perturbations up to the scale $\simeq 100 h^{-1} \mathrm{Mpc}$ determine the scale of the cosmic web in terms of void sizes. In contrast, waves of larger wavelengths do not influence the scale of but only amplify the web, leaving its scale unaffected. Thus, it is unclear, why the growth of the scale of the skeleton of the cosmic web with increasing wavelengths of density perturbations, stops at the perturbation scale $\simeq 100 \mathrm{~h}^{-1} \mathrm{Mpc}$. Has this some deeper physical meaning? That the change in the behaviour of the waves of different scale occurs in numerical simulations where only standard physics of the early Universe is included, implies that there is a simple answer.

For $h \approx 0.7$, this largest pattern scale is close to $R_{\mathrm{eq}} \equiv$ $a\left(t_{0}\right) \eta_{\mathrm{eq}}=2(\sqrt{2}-1)\left(c / H_{0}\right) \times \sqrt{\Omega_{\mathrm{rad}}} / \Omega_{\mathrm{m}} \approx 120 \mathrm{Mpc}$, which is the only scale characterising a primordial scale-free $\left(n_{\mathrm{s}} \approx\right.$ 1) spectrum of density perturbations multiplied by the scaledependent transfer function arising due to the transition from the radiation-dominated stage to the matter-dominated one, which occurred at $z=z_{\text {eq }} \approx 3200$ according to the most recent observational data (see Jarosik et al. 2011). Here the conformal time $\eta_{\mathrm{eq}}=\eta\left(z_{\mathrm{eq}}\right)=c \int_{z_{\mathrm{eq}}}^{\infty} \mathrm{d} z / H(z), H \equiv \dot{a}(t) / a(t)$ is the Hubble parameter, $t_{0}$ is the present time $\left(H\left(t_{0}\right)=H_{0}\right)$, and $\Omega_{\text {rad }}$ includes the contribution from three species of neutrino which all may be considered massless at the moment of matter-radiation energydensity equality $z=z_{\text {eq }}$. We note that $R_{\text {eq }}$ does not depend on $h$ if expressed in terms of $\Omega_{\mathrm{rad}} h^{2} \propto T_{\gamma}^{4}$ and $\Omega_{\mathrm{m}} h^{2}$, nor depend on the dark energy equation of state $w_{\mathrm{DE}}\left(w_{\mathrm{DE}}=-1\right.$ for a cosmological constant), only the present value of $\Omega_{\mathrm{m}}$ being important. Moreover, the expression for $R_{\text {eq }}$ remains the same for an open (negatively spatially curved) Universe in the absence of a cosmological constant.

Additional insight to this problem is given by simulations designed to understand the possible future of the development of the cosmic web. These simulations were performed by a number of authors (Loeb 2002; Nagamine \& Loeb 2003; Busha et al. 2005; Dünner et al. 2006, 2007; Hoffman et al. 2007; Krauss \& Scherrer 2007). In the early Universe, it is well known that the matter density $\Omega_{\mathrm{m}}$ dominated over the energy density. As time proceeded and the Universe expanded, the $\Omega_{\Lambda}$ term increased and caused the Universe to expand in an accelerating fashion from a redshift of $z \simeq 0.8$.

Our results for the absence of the growth of the scale of the cosmic web with the increase in the scale of density perturbations over $\simeq 100 h^{-1}$ Mpc can probably be explained as the result of the freezing of the web that started at recent redshifts, $1+z<\left(\left(1-\Omega_{\mathrm{m}}\right) / \Omega_{\mathrm{m}}\right)^{1 / 3}$ for an exact cosmological constant. Simulations of the future development of the web mentioned above support this interpretation. As stated by Hoffman et al. (2007) "in comoving coordinates the future large-scale structure will look like a sharpened image of the present structure: the skeleton of the cosmic web will remain the same, but clusters will be more isolated and the filaments will become thinner". Simulations by Nagamine \& Loeb (2003) and Hoffman et al. (2007) among others have shown that the mass evolution of bound objects such as clusters will stop. In the very distant future, all galaxies that are not bound to the Local Group (which consists basically of the merged Milky Way and M 31) will fade away while approaching the event horizon, as seen from an observer inside the Local Group. It remains to be investigated how a possible deviation of $w_{\mathrm{DE}}$ from -1 , if it exists, can affect the freezing and the properties of the cosmic web.

\section{Conclusions}

The basic conclusions of our study are as follows:

- The properties of the cosmic web depend strongly on density perturbations of various scales.

- Small-scale perturbations up to the scale $\simeq 8 h^{-1} \mathrm{Mpc}$ are responsible for the formation of galaxy and cluster type systems.

- Medium-scale perturbations of the scale $8 \ldots 64 h^{-1} \mathrm{Mpc}$ form the filamentary web inside and between the superclusters. They also contribute to the amplification of the systems formed by perturbations of smaller scales.

- The cosmic web with filamentary superclusters and voids is formed by the combined action of all perturbations up to the scale $\simeq 100 h^{-1} \mathrm{Mpc}$. The largest perturbations in this range determine the scale of the supercluster-void network.

- Perturbations of the largest scales $>100 h^{-1}$ Mpc modulate the richness of galaxy systems from clusters to superclusters, and make voids emptier.

Acknowledgements. We thank the anonymous referee for stimulating suggestions. The present study was supported by the Estonian Science Foundation grants No. 7146 and 8005, and by the Estonian Ministry for Education and Science grant SF0060067s08. The study has also been supported by ICRAnet through a professorship for Jaan Einasto, and by the University of Valencia (Vicerrectorado de Investigación) through a visiting professorship for Enn Saar and by the Spanish MEC projects "ALHAMBRA" (AYA2006-14056) and "PAU" (CSD2007-00060), including FEDER contributions. J.E., I.S. and E.T. thank Astrophysikalisches Institut Potsdam (using DFG-grant Mu 1020/15-1), where part of this study was performed. J.E. thanks also the Aspen Center for Physics and the Johns Hopkins University for hospitality where this project was started and continued. The simulation for the model L256 was calculated at the High Performance Computing Centre, University of Tartu. In plotting of density fields we used the SAOImage DS9 program. A.A.S. acknowledges the RESCEU hospitality as a visiting professor. He was also partially supported by the Russian Foundation for Basic Research grant No. 11-02-00643 and by the Scientific Programme "Astronomy" of the Russian Academy of Sciences. We thank the SDSS Team for the publicly available data releases. Funding for the SDSS and SDSS-II has been provided by the Alfred P. Sloan Foundation, the Participating Institutions, the National Science Foundation, the US Department of Energy, the National Aeronautics and Space Administration, the Japanese Monbukagakusho, the Max Planck Society, and the Higher Education Funding Council for England. The SDSS Web Site is http://www.sdss.org/. The SDSS is managed by the Astrophysical Research Consortium for the Participating Institutions. The Participating Institutions are the American Museum of Natural History, Astrophysical Institute Potsdam, University of Basel, University of Cambridge, Case Western Reserve University, University of Chicago, Drexel University, Fermilab, the Institute for Advanced Study, the Japan Participation Group, Johns Hopkins University, the Joint Institute for Nuclear Astrophysics, the Kavli Institute for Particle Astrophysics and Cosmology, the Korean Scientist Group, the Chinese Academy of Sciences (LAMOST), Los Alamos National Laboratory, the Max-Planck-Institute for Astronomy (MPIA), the Max-Planck-Institute for Astrophysics (MPA), New Mexico State University, Ohio State University, University of Pittsburgh, University of Portsmouth, Princeton University, the United States Naval Observatory, and the University of Washington.

\section{References}

Abazajian, K. N., Adelman-McCarthy, J. K., Agüeros, M. A., et al. 2009, ApJS, 182,543

Abell, G. O. 1958, ApJS, 3, 211

Abell, G. O., Corwin, Jr., H. G., \& Olowin, R. P. 1989, ApJS, 70, 1

Aragón-Calvo, M. A., Jones, B. J. T., van de Weygaert, R., \& van der Hulst, J. M. 2007, A\&A, 474, 315

Aragon-Calvo, M. A., van de Weygaert, R., Araya-Melo, P. A., Platen, E., \& Szalay, A. S. 2010a, MNRAS, 404, L89

Aragón-Calvo, M. A., van de Weygaert, R., \& Jones, B. J. T. 2010b, MNRAS, 408, 2163

Bagla, J. S., \& Prasad, J. 2006, MNRAS, 370, 993

Bagla, J. S., Prasad, J., \& Khandai, N. 2009, MNRAS, 395, 918

Bernardeau, F., Colombi, S., Gaztañaga, E., \& Scoccimarro, R. 2002, Phys. Rep., 367,1 
Bertschinger, E. 1995 [arXiv: astro-ph/9506070]

Bertschinger, E., \& Gelb, J. M. 1991, Comput. Phys., 5, 164

Bond, J. R., Szalay, A. S., \& Turner, M. S. 1982, Phys. Rev. Lett., 48, 1636

Bond, J. R., Kofman, L., \& Pogosyan, D. 1996, Nature, 380, 603

Bond, N. A., Strauss, M. A., \& Cen, R. 2010a, MNRAS, 406, 1609

Bond, N. A., Strauss, M. A., \& Cen, R. 2010b, MNRAS, 409, 156

Busha, M. T., Evrard, A. E., Adams, F. C., \& Wechsler, R. H. 2005, MNRAS, 363, L11

Colberg, J. M., Pearce, F., Foster, C., et al. 2008, MNRAS, 387, 933

Dünner, R., Araya, P. A., Meza, A., \& Reisenegger, A. 2006, MNRAS, 366, 803

Dünner, R., Reisenegger, A., Meza, A., Araya, P. A., \& Quintana, H. 2007, MNRAS, 376, 1577

Einasto, J., Jõeveer, M., \& Saar, E. 1980, MNRAS, 193, 353

Einasto, J., Klypin, A., \& Shandarin, S. 1983, in Early Evolution of the Universe and its Present Structure, ed. G. O. Abell, \& G. Chincarini, IAU Symp., 104, 265

Einasto, J., Saar, E., \& Klypin, A. A. 1986, MNRAS, 219, 457

Einasto, J., Einasto, M., \& Gramann, M. 1989, MNRAS, 238, 155

Einasto, J., Einasto, M., Gramann, M., \& Saar, E. 1991, MNRAS, 248, 593

Einasto, M., Einasto, J., Tago, E., Dalton, G. B., \& Andernach, H. 1994, MNRAS, 269, 301

Einasto, J., Einasto, M., Gottlöber, S., et al. 1997a, Nature, 385, 139

Einasto, M., Tago, E., Jaaniste, J., Einasto, J., \& Andernach, H. 1997b, A\&AS, 123,119

Einasto, M., Einasto, J., Tago, E., Müller, V., \& Andernach, H. 2001, AJ, 122, 2222

Einasto, J., Einasto, M., Saar, E., et al. 2006, A\&A, 459, L1

Einasto, J., Einasto, M., Tago, E., et al. 2007, A\&A, 462, 811

Einasto, J., Hutsi, G., Saar, E., et al. 2011, A\&A, 2011, A\&A, 531, A75

Erdoğdu, P., Lahav, O., Zaroubi, S., et al. 2004, MNRAS, 352, 939

Forero-Romero, J. E., Hoffman, Y., Gottlöber, S., Klypin, A., \& Yepes, G. 2009, MNRAS, 396, 1815

Gelb, J. M., \& Bertschinger, E. 1994, ApJ, 436, 467

Gottlöber, S., Łokas, E. L., Klypin, A., \& Hoffman, Y. 2003, MNRAS, 344, 715

Gramann, M. 1988, MNRAS, 234, 569

Gregory, S. A., \& Thompson, L. A. 1978, ApJ, 222, 784

Hahn, O., Porciani, C., Carollo, C. M., \& Dekel, A. 2007, MNRAS, 375, 489

Hoffman, Y., Lahav, O., Yepes, G., \& Dover, Y. 2007, J. Cosmology Astropart Phys., 10, 16

Jõeveer, M., \& Einasto, J. 1978, in Large Scale Structures in the Universe, ed. M. S. Longair, \& J. Einasto, IAU Symp., 79, 241

Jõeveer, M., Einasto, J., \& Tago, E. 1978, MNRAS, 185, 357

Jarosik, N., Bennett, C. L., Dunkley, J., et al. 2011, ApJS, 192, 14

Jones, B. J. T., van de Weygaert, R., \& Aragón-Calvo, M. A. 2010, MNRAS, 408,897

Kalinkov, M., \& Kuneva, I. 1995, A\&AS, 113, 451
Knebe, A., Kravtsov, A. V., Gottlöber, S., \& Klypin, A. A. 2000, MNRAS, 317, 630

Knebe, A., Green, A., \& Binney, J. 2001, MNRAS, 325, 845

Knollmann, S. R., \& Knebe, A. 2009, ApJS, 182, 608

Krauss, L. M., \& Scherrer, R. J. 2007, General Relativity and Gravitation, 39, 1545

Liivamägi, L. J., Tempel, E., \& Saar, E. 2010, A\&A, submitted [arXiv: 1012.1989]

Lindner, U., Einasto, J., Einasto, M., et al. 1995, A\&A, 301, 329

Lindner, U., Einasto, M., Einasto, J., et al. 1996, A\&A, 314, 1

Loeb, A. 2002, Phys. Rev. D, 65, 047301

Martel, H., \& Wasserman, I. 1990, ApJ, 348, 1

Martínez, V. J., \& Saar, E. 2002, Statistics of the Galaxy Distribution, ed. V. J. Martínez, \& E. Saar (Chapman \& Hall/CRC)

Melott, A. L., Einasto, J., Saar, E., et al. 1983, Phys. Rev. Lett., 51, 935

Nagamine, K., \& Loeb, A. 2003, New A, 8, 439

Oort, J. H. 1983, ARA\&A, 21, 373

Peebles, P. J. E. 2001, ApJ, 557, 495

Power, C., \& Knebe, A. 2006, MNRAS, 370, 691

Sheth, R. K., \& van de Weygaert, R. 2004, MNRAS, 350, 517

Sousbie, T., Pichon, C., Colombi, S., Novikov, D., \& Pogosyan, D. 2008, MNRAS, 383, 1655

Sousbie, T., Colombi, S., \& Pichon, C. 2009, MNRAS, 393, 457

Springel, V. 2005, MNRAS, 364, 1105

Springel, V., Yoshida, N., \& White, S. D. M. 2001, New A, 6, 79

Tago, E., Saar, E., Tempel, E., et al. 2010, A\&A, 514, A102

Tarenghi, M., Tifft, W. G., Chincarini, G., Rood, H. J., \& Thompson, L. A. 1978, in Large Scale Structures in the Universe, ed. M. S. Longair, \& J. Einasto, IAU Symp., 79, 263

Tegmark, M., Strauss, M. A., Blanton, M. R., et al. 2004, Phys. Rev. D, 69, 103501

Tempel, E., Saar, E., Liivamägi, L. J., et al. 2011, A\&A, 529, A53

Tully, R. B., \& Fisher, J. R. 1978, in Large Scale Structures in the Universe, ed. M. S. Longair, \& J. Einasto, IAU Symp., 79, 214

van de Weygaert, R., \& Platen, E. 2009 [arXiv: 0912 . 2997]

van de Weygaert, R., \& van Kampen, E. 1993, MNRAS, 263, 481

van de Weygaert, R., Aragon-Calvo, M. A., Jones, B. J. T., \& Platen, E. 2009 [arXiv:0912.3448]

von Benda-Beckmann, A. M., \& Müller, V. 2008, MNRAS, 384, 1189

White, S. D. M., Frenk, C. S., \& Davis, M. 1983, APJ, 274, L1

White, S. D. M., Frenk, C. S., Davis, M., \& Efstathiou, G. 1987, ApJ, 313, 505

Zeldovich, Y. B. 1970, A\&A, 5, 84

Zeldovich, Y. B. 1978, in Large Scale Structures in the Universe, ed. M. S. Longair, \& J. Einasto, IAU Symp., 79, 409

Zeldovich, Y. B., Einasto, J., \& Shandarin, S. F. 1982, Nature, 300, 407

Zucca, E., Zamorani, G., Scaramella, R., \& Vettolani, G. 1993, ApJ, 407, 470 